\title{
Role of Catheter Ablation for Ventricular Arrhythmias in Brugada Syndrome
}

\author{
Ronpichai Chokesuwattanaskul ${ }^{1} \cdot$ Koonlawee Nademanee ${ }^{1,2,3,4}$
}

Accepted: 17 February 2021 / Published online: 24 April 2021

(C) The Author(s) 2021

\begin{abstract}
Purpose of Review To discuss the role of catheter ablation in treating life-threatening ventricular arrhythmias associated with Brugada syndrome $(\mathrm{BrS})$, by presenting recent findings of $\mathrm{BrS}$ arrhythmogenic substrate, mechanisms underlying ventricular arrhythmias, and how they can be treated with catheter ablation.

Recent Findings Almost three decades ago when the clinical entity of Brugada syndrome (BrS) was described in patients who had abnormal coved-type ST elevation in the right precordial EKG leads in patients who had no apparent structural heart disease but died suddenly from ventricular fibrillation. Since its description, the syndrome has galvanized explosive research in this field over the past decades, driving major progress toward better understanding of BrS, gaining knowledge of the genetic pathophysiology and risk stratification of BrS, and creating significant advances in therapeutic modalities. One of such advances is the ability for electrophysiologists to map and identify the arrhythmogenic substrate sites of BrS, which serve as good target sites for catheter ablation. Subsequently, several studies have shown that catheter ablation of these substrates normalizes the Brugada ECG pattern and is very effective in eliminating these substrates and preventing recurrent VF episodes.

Summary Catheter ablation has become an important addition for treatment of symptomatic BrS patients with recurrent VT/VF episodes.
\end{abstract}

Keywords Brugada $\cdot$ Catheter ablation $\cdot$ Sudden cardiac death $\cdot$ Ventricular arrhythmia

\section{Introduction}

Brugada syndrome $(\mathrm{BrS})$ was first described almost 3 decades ago and was first considered a primary electrical disease manifesting as life-threatening polymorphic ventricular

This article is part of the Topical Collection on Invasive Electrophysiology and Pacing

Koonlawee Nademanee wee@pacificrimep.com

Ronpichai Chokesuwattanaskul

dr_ronpichai_c@yahoo.com

1 Faculty of Medicine, Division of Cardiovascular Medicine, King Chulalongkorn Memorial Hospital, Chulalongkorn University, Bangkok 10330, Thailand

2 Bumrungrad Hospital, Bangkok, Thailand

3 Pacific Rim Electrophysiology Research Institute, Bangkok, Thailand

4 Pacific Rim Electrophysiology Research Institute, Las Vegas, Nevada, USA tachycardia/ventricular fibrillation (VT/VF) causing sudden cardiac death (SCD) [1]. BrS is characterized by right precordial lead coved-type ST elevation (Fig. 1) with no overt structural heart disease or secondary factors (e.g., myocardial ischemia, electrolyte imbalance) [2•]. During the first decade, two consensus statements laid out diagnostic criteria, risk stratification, and preliminary genetic aspects of the syndrome $[2 \bullet, 3 \bullet]$. Over the last 2 decades, the explosion of seminal research studies shed light on underlying electrophysiological mechanisms of the syndrome - a complex interplay between genetics, myocardial substrate abnormalities, and channelopathy_leading to the development of a new therapeutic modality, catheter ablation of $\mathrm{BrS}$ substrates $[4 \cdot, 5,6]$. The purpose of this review is to discuss the role of catheter ablation in treating life-threatening ventricular arrhythmias associated with BrS, not other aspects of the syndrome. To learn more about other aspects of $\mathrm{BrS}$, we recommend reading several excellent reviews $[4 \cdot, 5,6]$. Herein, we will describe recent findings of $\mathrm{BrS}$ arrhythmogenic substrate, mechanisms underlying ventricular arrhythmias, and how they can be treated with catheter ablation. 


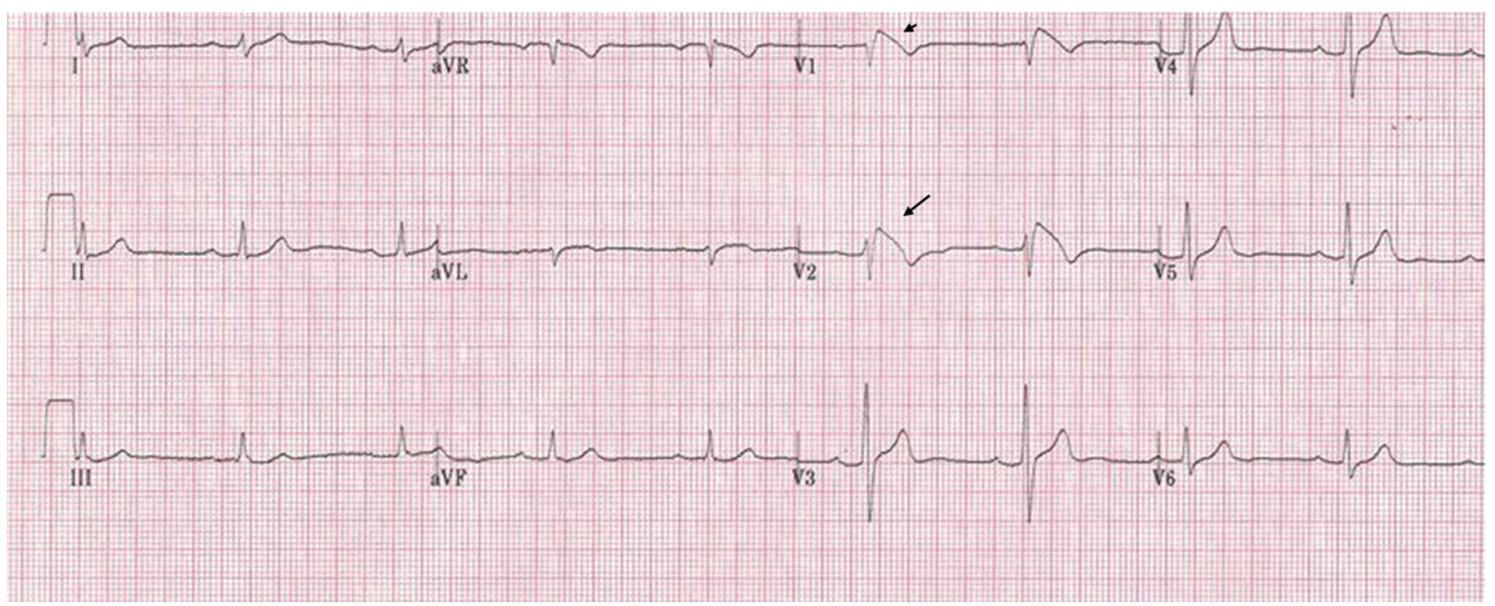

Fig. 1 A 12 lead EKG of a BrS patient showing type I Brugada ECG pattern with a signature curved-type ST elevation in V1 and V2 (arrows).

\section{BrS Arrhythmogenic Substrates}

It was recently established that right ventricular (RV) epicardium, especially the RV outflow tract (RVOT), is the primary arrhythmogenic substrate site of BrS. Here, abnormal electrograms, defined by low voltage $(\leq 1 \mathrm{mV})$, prolonged duration (>120 ms), and fractionated potential beyond QRS complex, are omnipresent [7]. These RVOT sites that harbor such abnormal fragmented electrograms were found to have marked epicardial and interstitial myocardial fibrosis, from both biopsy studies of symptomatic $\mathrm{BrS}$ patients who underwent direct open thoracotomy ablation for treatment of recurrent VF, and autopsy studies of SCD victims who had a BrS family history. The RVOT sites of the heart were also found to have a diminished connexin- 43 expression, resulting in a decrease in gap junction protein connexin- 43 when compared to the matched control subjects $[8 \cdot \bullet]$. The presence of myocardial and epicardial fibrosis in the $\mathrm{RV}$ epicardium of BrS patients likely create a milieu [9], as demonstrated by the study of De Bakker et al. [10], where an electrical impulse must travel in a slow zig-zag conduction manner through the fibrotic areas producing latefractionated electrogram on RVOT epicardial electrograms, reentrant polymorphic VT/VF, and corresponding BrS type 1 electrocardiogram (ECG) phenotype.

Of significance, our group was the first to show that when radiofrequency ablations were delivered on these fibrotic areas with fractionated electrograms in the RVOT/RV epicardium, Brugada ECG patterns were normalized and effective in preventing recurrent spontaneous VF episodes as well as inducible VF by programmed electrical stimulation [7, 8••]. These observations clearly provide proof that underlying myopathic changes along with their electrophysiological abnormalities likely result in heterogeneity of impulse conduction, conduction delay, and dispersion RV/RVOT epicardium causing reentry as the main electrophysiological mechanism for $\mathrm{VF}$ and the signature EKG abnormality in BrS.

\section{BrS Treatment Before Catheter Ablation}

During the first 2 decades of discovering the syndrome, several studies revealed that symptomatic BrS patients - patients who survived previous cardiac arrests, patients with history of unknown syncope/seizure, or patients with observed agonal respiration during sleep - are at high risk for having recurrent VF episodes [11-13]. An implantable cardioverterdefibrillator (ICD) is unequivocally recommended as firstline therapy to prevent SCD in these high-risk patients [3•]. In contrast, there have been no clearly established recommendations for treatment of asymptomatic BrS patients [3•, 14-16]. This was largely due to the lack of strong predictors of VF in the asymptomatic group. Recent studies have reported low but non-negligible arrhythmic events in asymptomatic BrS patients, about a $0.5-1.2 \%$ annual incidence, meaning a $12 \%$ malignant arrhythmia event at 10 -year follow-up [6, 7, $8 \bullet \bullet]$. Some studies recommended that electrophysiology studies be performed to help risk-stratify and decide if ICD is indicated [14-21]. Also, ICD treatment in asymptomatic $\mathrm{BrS}$ patients was found to be associated with significant complications, i.e., cardiac implantable electronic device (CIED) implantation, inappropriate ICD therapies, pocket infection, lead fracture, and chronic lead issues [22-24]. As a result, the current guideline does not recommend ICD treatment for asymptomatic patients [3•].

Quinidine is the only anti-arrhythmic drug found to be efficacious in preventing recurrent VF $[25,26]$. However, universal inaccessibility, unwanted adverse effects, and patient non-compliance preclude widespread long-term use in most patients [26]. Furthermore, there are scarcity of studies that have a large sample size or randomized clinical trials to critically evaluate safety and efficacy of the drug in symptomatic BrS.

ICD therapy alone does not prevent recurrent VF episodes; the device is merely designed to rescue patients once VF 
occurs [27]. Numerous BrS patients have suffered from CIED-related issues such as frequent ICD shocks from VT/ VF episodes. In the past, many BrS patients had suffered VF storms despite concomitant quinidine treatment that became perilous for such patients to receive intensive care treatment that included isoproterenol infusion, deep sedation, and other anti-arrhythmic drugs including amiodarone. A significant number of these patients succumbed from refractory VF despite medical treatment or suffered hundreds of multiple ICD shocks; a few patients had to undergo cardiac transplantation as a life-saving measure [9]. In short, during the first 2 decades of $\mathrm{BrS}$, there were very limited treatment options for symptomatic patients. Fortunately, ablation of the BrS substrate was developed over the last decade for treatment of the subset of VF storms as well as for symptomatic BrS patients [7].

\section{Catheter Ablation Treatment}

Haïssaguerre and colleagues [29] were the first to attempt catheter ablation to treat $\mathrm{BrS}$ patients with recurrent VF. They utilized a VF-trigger ablation approach in symptomatic BrS patients. However, this VF trigger-targeted approach is limited because spontaneous VF-trigger premature ventricular contractions (PVCs) rarely present during the mapping procedure. This poses a major challenge for operators who try to identify the focus of these PVCs triggers, thus precluding its widespread use for treatment of symptomatic BrS.

Subsequently, our group was the first to report the feasibility of epicardial ablations of the VF substrates and that the procedure, utilized in 9 symptomatic BrS patients with recurrent VF episodes, was indeed safe and effective. Since our initial report [7], several studies worldwide have confirmed our findings of epicardial ablation of VF substrates, and the procedure has been increasingly utilized for treatment of symptomatic $\mathrm{BrS}$ patients [30-34].

\section{How to Map Substrate in BrS Patients}

BrS arrhythmogenic substrate sites have unique electrogram characteristics enabling operators to identify them precisely and easily. To map substrate, one must gain access to pericardial space for epicardial mapping. Both ventricular endocardium and epicardium are mapped with electroanatomical mapping. The mapping system and catheter should be selected based on operators' preference provided they render high-density electroanatomic mapping. In addition to mapping, image-integration tools, such as CartoUnivu and non-invasive electrocardiographic imaging (ECGI), are beneficial because they [1] provide detailed anatomy and its relationship to the substrate site both during sinus rhythm and during VF [2], identify substrate sites, and [3] prevent collateral damage during radiofrequency application in the proximity of the coronary artery. An example of the map is shown in Fig. 2 which was obtained from a BrS patients with frequent recurrent VF episodes necessitating multiple ICD shocks. The map delineates the targeted area for ablation tagged by the abnormal low-voltage fractionated signals, which comprises multiple components with more than one distinct component, with $>20 \mathrm{~ms}$ isoelectric segments between peaks of individual components, and long duration $(\geq 70 \mathrm{~ms})$ or late potentials that are beyond the end of QRS complex [28•]. After the ablation, the patient's EKG has been normalized (Fig. 3) and free of recurrent VF for over 3 years.

\section{Role of Sodium Channel Blockers in Exposing Subtle Substrate Sites}

In our initial report, we performed epicardial RVOT ablation of the first 9 patients without using sodium channel blockade, ajmaline, during the mapping since at that time the Institution Review Board (IRB) did not approve utilization of the drug during the procedure [7]. Subsequently, after the safety of the procedure was established, the IRB granted approval of using ajmaline to further define the substrate sites. Indeed, we found that ajmaline infusion could unmask the subtle substrate sites and further increase the target area to almost twice the size, from 14.3 to $20.4 \mathrm{~cm} \mathrm{[2•].} \mathrm{A} \mathrm{few} \mathrm{patients} \mathrm{from} \mathrm{our} \mathrm{initial}$ report experienced recurrence caused by incomplete substrate elimination. These patients required repeat ablation; during this repeated procedure, more substrate areas that were missed during the first procedure were exposed by ajmaline infusion and guided successful ablations [28.]. Pappone and colleagues also reported similar findings with both flecainide and ajmaline that these sodium channel blockades were beneficial in aiding $\mathrm{BrS}$ substrates [30,31]. As a result, a sodium channel blocker has now been included in a mapping protocol to identify all possible substrates.

In the past, endocardial ablation of the substrate sites in the $\mathrm{RV}$ was also introduced with promising results [35]. However, the long-term results could not be reproduced among clinical studies. Talib et al. conducted a study demonstrating a stepwise approach of endocardial ablation by searching the VF-triggering area followed by VF substrate ablation endocardial ablation [36]. While they had modest success with VF-trigger ablation, the endocardial approach for elimination of VF substrates yielded a low success rate. This finding was not surprising because virtually all studies found that almost all substrate sites are located in the RV epicardium and not in the corresponding endocardial sites. Thus, it has been our assertion that BrS substrate ablation cannot be carried out successfully without epicardial substrate ablation [37]. 


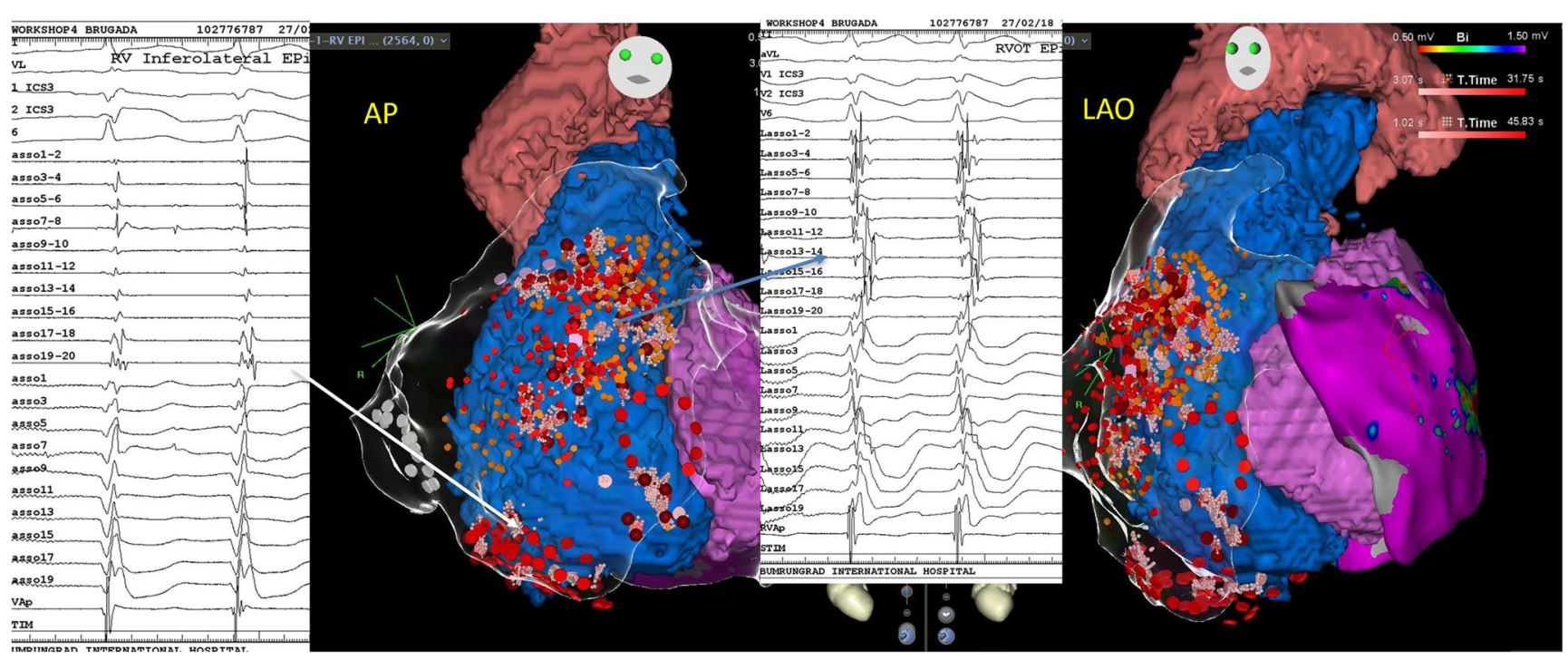

Fig. 2 An example of electroanatomic mapping of VF substrates from a BrS patient who had recurrent VF episodes triggering multiple ICD discharges. The electroanatomical maps display areas of fractionated electrograms during sinus rhythm recorded with a 20-pole Lasso catheter in the RV epicardium. The maps are displayed in the anterior posterior (AP) and the left anterior oblique (LAO). Abnormal fractionated potential over the RVOT, RV body, and RV inferolateral are shown in the first and the third insets organized in the following fashion: The top 4 tracings are $\mathrm{L} 1, \mathrm{AVL}, \mathrm{V} 1$, and $\mathrm{V} 2$ positioned at the 3rd intercostal space $\left(\mathrm{V} 1 \mathrm{ICS}_{3}\right.$ and $\mathrm{V} 2 \mathrm{ICS}_{3}$ respectively) followed with 10 bipolar recordings from the Lasso catheter and the Unipolar recordings from the Lasso poles

\section{Ablation Protocol and Endpoints}

An irrigated-tip catheter with contact force measurement should be used to apply force for $\geq 5 \mathrm{~g}$ before radiofrequency energy is delivered. The power is titrated from 20 to $50 \mathrm{~W}$ to achieve $\geq 10$ ohms impedance reduction from baseline. This force and power should provide sufficient acute elimination effect on the target area. Effective lesions were confirmed by rapid disappearance of late/fractionated components and a decline in recorded signal amplitude. The endpoint is a complete elimination of substrate
$1,3,5,7,9,11,13,15,17$, and 19). The first inset is the Lasso catheter recordings from the RV inferior epicardium and exhibits fractionated signals from the bipolar recordings from the Lasso 17-18 and 19-20. The third inset is the lasso catheter recordings from the RVOT epicardium and show abnormal fractionated late electrograms in all bipolar and unipolar recordings. The red dots on the electroanatomical maps represent VF substrates that harbor abnormal fractionated electrograms. Small pink dots are the areas that RF application were delivered. Note that this patient had an extensive epicardial substrates from the RVOT, RV body, and RV inferior wall

identified after ajmaline infusion over the RVOT and its vicinity. Non-inducible VT/VF and disappearance of the BrS ECG pattern can be used as an adjunct endpoint of ablation.

\section{Efficacy and Safety of Catheter Ablation for BrS}

We have performed ablation on 54 symptomatic BrS patients (median age, 37 years; 1 female). All had ICD for cardiac arrest ( $N=48)$ or syncope $(N=6)$. All underwent a percutaneous epicardial substrate ablation with electroanatomical mapping.
Fig. 3 Normalization of Brugada ECG pattern in V1 and V2 after ablation. Both EKGs were taken after ajmaline administration (50 $\mathrm{mg})$

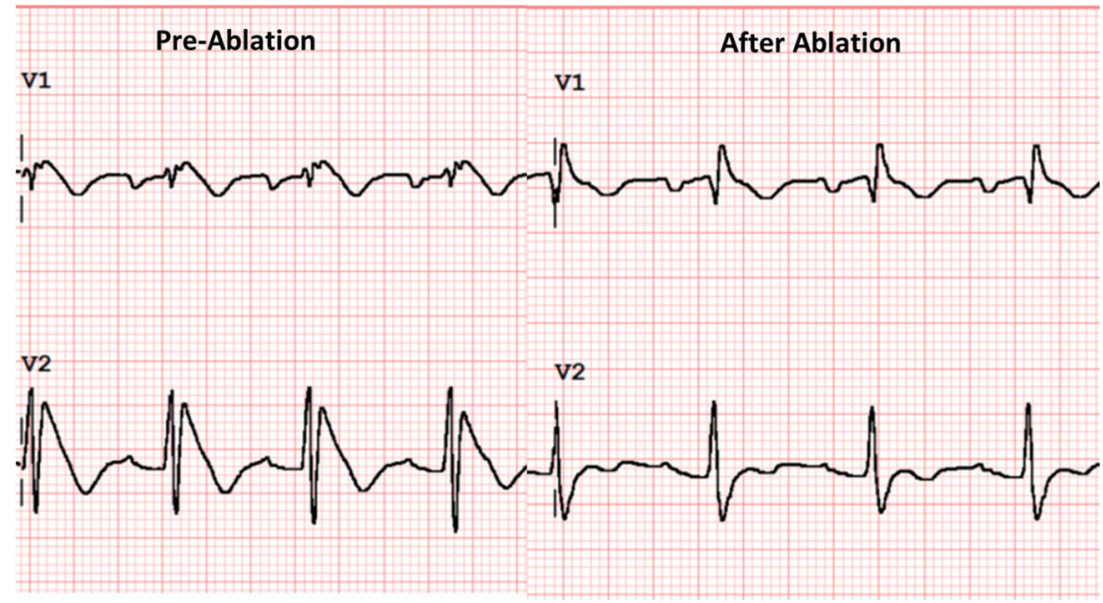


All patients had BrS substrates in the RVOT epicardium but 25 (47\%) also had substrates elsewhere: in the RV exhibiting latefractionated potentials extended to the RV body $(N=14)$, inferior $\mathrm{RV}(N=4)$, and both body and inferior $\mathrm{RV}(N=7)$, respectively. After the first catheter ablation, 45 of the $54(85 \%)$ became VFfree. After a repeat CA, 52 patients $(96 \%)$ became VF-free without anti-arrhythmic drugs (follow-up period $=32 \pm 30$ months; $p$ $<0.01)$. The main predictor of VF recurrence necessitating repeat ablation is the continuing presence of Brugada ECG pattern and a combined $\mathrm{BrS}$ and early repolarization (ER). No major complications occurred, except 1 hemopericardium and 1 pericarditis.

Recent studies from other institutions have confirmed our findings that $\mathrm{BrS}$ arrhythmogenic substrates are present in the $\mathrm{RV} / \mathrm{RVOT}$ epicardium in virtually all symptomatic BrS patients, and catheter ablations of these substrates are safe and effective for prevention of VF recurrences in these patients.

\section{Combined Syndrome of BrS and ERS}

In our patient population in Southeast Asia, approximately 20\% have a combined Brugada and early repolarization EKG pattern either in the inferior leads (L II, III, and AVF) or in the lateral leads $\left(\mathrm{V}_{4}-\mathrm{V}_{6}\right)$ or in both inferior and lateral leads. Several studies in the past decade show that these overlapping features of $\mathrm{BrS}$ and ER syndrome (ERS) increase risk of recurrent VT/VF much higher than that pose by $\mathrm{BrS}$ alone [38-40]. As indicated above, this subset also presents a daunting task for catheter ablation because the VF recurrent rate, after the first session of ablation, is higher than that of $\mathrm{BrS}$ alone. Fortunately, we have now gained more experience in performing mapping and ablation of this subset as we have learned that the combined BrS and ERS patients have larger substrates than $\mathrm{BrS}$ alone; in addition to the RVOT substrate sites, the combined syndrome patients commonly have substrates at the RV inferior wall and RV inferolateral areas closed to the tricuspid annulus [41•]. With this knowledge, we have been more proficient in eliminating these substrates yielding better long-term outcomes than the initial encounter with the combined syndrome.

\section{Conclusion and Future Direction}

Better understanding of pathophysiologic mechanisms and underlying pathology of $\mathrm{BrS}$ has enabled electrophysiologists to map and identify these substrate sites, which are exclusively present in the RV/RVOT epicardium. These sites serve very well as target areas for catheter ablation. Catheter ablation is effective in eliminating these substrates and preventing recurrent VF episodes. Thus, catheter ablation is an important addition for treatment of symptomatic BrS patients with recurrent VT/VF episodes. In many instances, ablation becomes the sole therapeutic option, especially in the case of drug-resistant $\mathrm{BrS}$ or in places where quinidine is not available.
Three key questions need to be answered in the future: (1) Can ablation replace ICD as first-line treatment if all BrS substrates are eliminated? (2) Should asymptomatic BrS patients be treated with ablation? (3) Would extensive substrate ablation over the RV predispose to scar-related reentry monomorphic VT and deterioration of RV function in the long-term? We hope to answer these questions through future randomized clinical trials. Currently, the ablation in $\mathrm{BrS}$ for the prevention of $\mathrm{VF}$ (BRAVE, Clinicaltrials.gov identifier NCT02704416) study is being conducted; results, available in the next few years, should demonstrate which patients are suitable to be treated by ablation as a curable approach without ICD with long-lasting beneficial effects. At present, we believe that epicardial ablation should be considered the treatment of choice in $\mathrm{BrS}$ patients who experience recurrent VF episodes.

Author Contribution All authors had access to the data and a role in writing the manuscript.

Funding This work is supported by The National Research Council of Thailand (grant number 2558-114), a Grant-in Aid by Bumrungrad Hospital, and funding support from the Center of Excellence in Arrhythmia Research Chulalongkorn University.

\section{Declarations}

Conflict of Interest Dr. Chokesuwattanaskul declare that he has no conflict of interest. Dr. Nademanee has royalty income and consultant honoraria from Biosense Webster, Inc. and research grants from Biosense Webster, Inc. and Medtronic Inc.

Human and Animal Rights and Informed Consent This article does not contain any studies with human or animal subjects performed by any of the authors.

Open Access This article is licensed under a Creative Commons Attribution 4.0 International License, which permits use, sharing, adaptation, distribution and reproduction in any medium or format, as long as you give appropriate credit to the original author(s) and the source, provide a link to the Creative Commons licence, and indicate if changes were made. The images or other third party material in this article are included in the article's Creative Commons licence, unless indicated otherwise in a credit line to the material. If material is not included in the article's Creative Commons licence and your intended use is not permitted by statutory regulation or exceeds the permitted use, you will need to obtain permission directly from the copyright holder. To view a copy of this licence, visit http://creativecommons.org/licenses/by/4.0/.

\section{References}

Papers of particular interest, published recently, have been highlighted as:

- Of importance

•- Of major importance

1. Brugada P, Brugada J. Right bundle branch block, persistent ST segment elevation and sudden cardiac death: a distinct clinical and 
electrocardiographic syndrome. A multicenter report. J Am Coll Cardiol. 1992;20(6):1391-6.

2. Wilde AA, Antzelevitch C, Borggrefe M, Brugada J, Brugada R, Brugada $\mathrm{P}$, et al. Proposed diagnostic criteria for the Brugada syndrome: consensus report. Circulation. 2002;106:2514-9 The first consensus report that has created the criteria of $\mathrm{BrS}$ diagnosis.

3. Antzelevitch C, Brugada P, Borggrefe M, Brugada J, Brugada R, Corrado D, et al. Brugada syndrome: report of the second consensus conference: endorsed by the Heart Rhythm Society and the European Heart Rhythm Association. Circulation. 2005;111:65970 The second consensus report that has expanded the diganostic criteria, risk stratification, and management of $\mathrm{BrS}$.

4. Priori SG, Wilde AA, Horie M, Cho Y, Behr ER, Berul C, et al. Executive summary: HRS/EHRA/APHRS expert consensus statement on the diagnosis and management of patients with inherited primary arrhythmia syndromes. Europace. 2013;15(10):1389-406 An expert consensus of how one best diagnose and manage BrS.

5. Brugada J, Campuzano O, Arbelo E, Sarquella-Brugada G, Brugada R. Present status of Brugada syndrome. J Am Coll Cardiol. 2018;72(9):1046-59.

6. Veerakul G, Nademanee K. Brugada syndrome: two decades of progress. Circ J. 2012;76:2713-22.

7. Nademanee K, Veerakul G, Chandanamattha P, Chaothawee L, Ariyachaipanich A, Jirasirirojanakorn K, et al. Prevention of ventricular fibrillation episodes in Brugada syndrome by catheter ablation over the anterior right ventricular outflow tract epicardium. Circulation. 2011;123(12):1270-9.

8.• Nademanee K, Raju H, de Noronha SV, Papadakis M, Robinson L, Rothery S, et al. Fibrosis, connexin-43, and conduction abnormalities in the Brugada syndrome. J Am Coll Cardiol. 2015;66(18): 1976-86 The first paper to correlate myocardial fibrosis, arrhythmogenic substrates that harbor abnormal fractionated electrograms and the beneficial effects of ablation over these fibrotic areas

9. Coronel R, Casini S, Koopmann TT, Wilms-Schopman FJ, Verkerk $\mathrm{AO}$, de Groot JR, et al. Right ventricular fibrosis and conduction delay in a patient with clinical signs of Brugada syndrome: a combined electrophysiological, genetic, histopathologic, and computational study. Circulation. 2005;112:2769-77.

10. de Bakker JM, Wittkampf FH. The pathophysiologic basis of fractionated and complex electrograms and the impact of recording techniques on their detection and interpretation. Circ Arrhythm Electrophysiol. 2010;3:204-13.

11. Priori SG, Gasparini M, Napolitano C, Della Bella P, Ottonelli AG, Sassone B, et al. Risk stratification in Brugada syndrome: results of the PRELUDE (PRogrammed ELectrical stimUlation preDictive valuE) registry. J Am Coll Cardiol. 2012;59:37-45.

12. Sieira J, Conte G, Ciconte G, de Asmundis C, Chierchia GB, Baltogiannis G, et al. Prognostic value of programmed electrical stimulation in Brugada syndrome: 20 years experience. Circ Arrhythm Electrophysiol. 2015;8(4):777-84

13. Brugada J, Brugada R, Antzelevitch C, Towbin J, Nademanee K, Brugada P. Long-Term Follow-up of individuals with the electrocardiographic pattern of right bundle-branch block and ST-segment elevation in precordial leads V1 to V3. Circulation. 2002;105:78.

14. Sieira J, Ciconte G, Conte G, Chierchia GB, de Asmundis C, Baltogiannis G, et al. Asymptomatic Brugada syndrome: clinical characterization and long-term prognosis. Circ Arrhythm Electrophysiol. 2015;8(5):1144-50.

15.•P Priori SG, Blomstrom-Lundqvist C. European Society of Cardiology Guidelines for the management of patients with ventricular arrhythmias and the prevention of sudden cardiac death summarized by co-chairs. Eur Heart J. 2015;36(41):2757-9 This paper provides the evidence-based guidelines for management and prevention of Brugada syndrome.
16. Veerakul G, Camblock J, Schwab M, Nademanee K. Low mortality rate among asymptomatic Brugada syndrome patients: a multicenter control-randomized study comparing ICD-versus no-ICD treatment. Circulation. 2008;118:S 982.

17. Probst V, Veltmann C, Eckardt L, Meregalli PG, Gaita F, Tan HL, et al. Long-term prognosis of patients diagnosed with Brugada syndrome: results from the FINGER Brugada Syndrome Registry. Circulation. 2010;121:635-43.

18. Kamakura S, Ohe T, Nakazawa K, Aizawa Y, Shimizu A, Horie M, et al. Long-term prognosis of probands with Brugada-pattern STelevation in leads V1-V3. Circ Arrhythm Electrophysiol. 2009;2: 495-503.

19. Paul M, Gerss J, Schulze-Bahr E, Wichter T, Vahlhaus C, Wilde $\mathrm{AA}$, et al. Role of programmed ventricular stimulation in patients with Brugada syndrome: a meta-analysis of worldwide published data. Eur Heart J. 2007;28:2126-33.

20. Eckardt L, Probst V, Smits JP, Bahr ES, Wolpert C, Schimpf R, et al. Long-term prognosis of individuals with right precordial STsegment-elevation Brugada syndrome. Circulation. 2005;111:25763.

21. Takagi M, Yokoyama Y, Aonuma K, Aihara N, Hiraoka M, Investigators JIVFSJ-I. Clinical characteristics and risk stratification in symptomatic and asymptomatic patients with brugada syndrome: multicenter study in Japan. J Cardiovasc Electrophysiol. 2007;18:1244-51.

22. Sacher F, Probst V, Iesaka Y, Jacon P, Laborderie J, Mizon-Gérard $\mathrm{F}$, et al. Outcome after implantation of a cardioverter-defibrillator in patients with Brugada syndrome: a multicenter study. Circulation. 2006;114:2317-24.

23. Sarkozy A, Boussy T, Kourgiannides G, Chierchia GB, Richter S, De Potter T, et al. Long-term follow-up of primary prophylactic implantable cardioverter- defibrillator therapy in Brugada syndrome. Eur Heart J. 2007:28:334-44.

24. Steven D, Roberts-Thomson KC, Inada K, Seiler J, Koplan BA, Tedrow UB, et al. Long-term follow-up in patients with presumptive Brugada syndrome treated with implanted defibrillators. J Cardiovasc Electrophysiol. 2011;22:1115-9.

25. Shenthar J, Chakali SS, Acharya D, Parvez J, Banavalikar B. Oral quinine sulfate for the treatment of electrical storm and prevention of recurrent shocks in Brugada syndrome after failed cilostazol therapy. HeartRhythm Case Rep. 2017;3(10):470-4.

26. Viskin S, Wilde AA, Guevara-Valdivia ME, Daoulah A, Krahn $\mathrm{AD}$, Zipes DP, et al. Quinidine, a life-saving medication for Brugada syndrome, is inaccessible in many countries. J Am Coll Cardiol. 2013;61(23):2383-7.

27. Nademanee K, Veerakul G, Mower M, Likittanasombat K, Krittayapong R, Bhuripanyo K, et al. Defibrillator Versus betaBlockers for Unexplained Death in Thailand (DEBUT): a randomized clinical trial. Circulation. 2003;107:2221-6.

28. Nademanee K, Hocini M, Haïssaguerre M. Epicardial substrate ablation for Brugada syndrome. Heart Rhythm. 2017;14(3):45761 This paper gives an overview and rationale of catheter ablation of BrS.

29. Haissaguerre M, Extramiana F, Hocini M, Cauchemez B, Jais P, Cabrera JA, et al. Mapping and ablation of ventricular fibrillation associated with long-QT and Brugada syndromes. Circulation. 2003;108(8):925-8.

30. Brugada J, Pappone C, Berruezo A, Vicedomini G, Manguso F, Ciconte $\mathrm{G}$, et al. Brugada syndrome phenotype elimination by epicardial substrate ablation. Circ Arrhythm Electrophysiol. 2015;8: 1373-81.

31. Pappone C, Brugada J, Vicedomini G, et al. Electrical substrate elimination in 135 consecutive patients with Brugada syndrome. Circ Arrhythm Electrophysiol. 2017;10:e005053.

32. Chung FP, Raharjo SB, Lin YJ, Chang SL, Lo LW, Hu YF, et al. A novel method to enhance phenotype, epicardial functional 
substrates, and ventricular tachyarrhythmias in Brugada syndrome. Heart Rhythm. 2017;14:508-17.

33. Fernandes GC, Fernandes A, Cardoso R, Nasi G, Rivera M, Mitrani $\mathrm{RD}$, et al. Ablation strategies for the management of symptomatic Brugada syndrome: a systematic review. Heart Rhythm. 2018;15: 1140-7.

34. Zhang P, Tung R, Zhang Z, Sheng X, Liu Q, Jiang R, et al. Characterization of the epicardial substrate for catheter ablation of Brugada syndrome. Heart Rhythm. 2016;13:2151-8.

35. Sunsaneewitayakul B, Yao Y, Thamaree S, Zhang S. Endocardial mapping and catheter ablation for ventricular fibrillation prevention in Brugada syndrome. J Cardiovasc Electrophysiol. 2012;23:S10 6.

36. Talib AK, Takagi M, Shimane A, Nakano M, Hayashi T, Okajima $\mathrm{K}$, et al. Efficacy of endocardial ablation of drug-resistant ventricular fibrillation in Brugada syndrome: long-term outcome. Circ Arrhythm Electrophysiol. 2018;11(8):e005631.

37. Nademanee K, Haïssaguerre M. Endocardial ablation approach for Brugada syndrome: an important first step or a Quixotic quest. Circ Arrhythm Electrophysiol. 2018;11(8):e0006675.
38. Tokioka K, Kusano KF, Morita H, Miura D, Nishii N, Nagase S, et al. Electrocardiographic parameters and fatal arrhythmic events in patients with Brugada syndrome: combination of depolarization and repolarization abnormalities. J Am Coll Cardiol. 2014;63: 2131-8.

39. Sarkozy A, Chierchia GB, Paparella G, et al. Inferior and lateral electrocardiographic repolarization abnormalities in Brugada syndrome. Circ Arrhythm Electrophysiol. 2009;2:154-40.

40. Letsas KP, Sacher F, Probst V, et al. Prevalence of early repolarization pattern in inferolateral leads in patients with Brugada syndrome. Heart Rhythm. 2008;5:1685-9.

41. Nademanee K, Haïssaguerre M, Hocini, et al. Mapping and ablation of ventricular fibrillation associated with early repolarization syndrome. Circulation. 2019;140:1477-90 The first report of catheter ablation of the $\mathbf{J}$ wave syndrome substrates that includes the combined BrS and ERS.

Publisher's Note Springer Nature remains neutral with regard to jurisdictional claims in published maps and institutional affiliations. 